\title{
Notification List Notification that new names and new combinations
have appeared in volume 60 , part 7 , of the IJSEM
}

Correspondence

Jean Euzéby

email address can be found at

www.bacterio.net
This listing of names published in a previous issue of the IJSEM is provided as a service to bacteriology to assist in the recognition of new names and new combinations. This procedure was proposed by the Judicial Commission [Minute 11(ii), Int J Syst Bacterio/ 41 (1991), p. 185]. The names given herein are listed according to the Rules of priority (i.e. page number and order of valid publication of names in the original articles). Taxonomic opinions included in this List (i.e. the creation of synonyms or the emendation of circumscriptions) cannot be considered as validly published nor, in any other way, approved by the International Committee on Systematics of Prokaryotes and its Judicial Commission.

\begin{tabular}{|c|c|c|}
\hline Name/author(s): & Proposed as: & Page no. \\
\hline Corynebacterium pilbarense Aravena-Roman et al. 2010 & sp. nov. & 1486 \\
\hline Brevundimonas basaltis Choi et al. 2010 & sp. nov. & 1490 \\
\hline Paenochrobactrum gallinarii Kämpfer et al. 2010 & sp. nov. & 1496 \\
\hline $\begin{array}{l}\text { Paenochrobactrum glaciei (Romanenko et al. 2008) Kämpfer et al. } 2010 \text { (basonym } \\
\text { Pseudochrobactrum glaciei Romanenko et al. 2008) }\end{array}$ & comb. nov. & 1497 \\
\hline Streptomyces iranensis Hamedi et al. 2010 & sp. nov. & 1508 \\
\hline Koreibacter Lee and Lee 2010 & gen. nov. & 1513 \\
\hline Koreibacter algae Lee and Lee 2010 & sp. nov. & 1513 \\
\hline Actinomyces timonensis Renvoise et al. 2010 & sp. nov. & 1520 \\
\hline Stenotrophomonas ginsengisoli Kim et al. 2010 & sp. nov. & 1525 \\
\hline Enterorhabdus Clavel et al. 2009 emend. Clavel et al. 2010 & emend.* & 1530 \\
\hline Lysobacter soli Srinivasan et al. 2010 & sp. nov. & 1544 \\
\hline Ochrobactrum ciceri Imran et al. 2010 & sp. nov. & 1552 \\
\hline Rhodocytophaga Anandham et al. 2010 & gen. nov. & 1557 \\
\hline Rhodocytophaga aerolata Anandham et al. 2010 & sp. nov. & 1557 \\
\hline Enterobacter arachidis Madhaiyan et al. 2010 & sp. nov. & 1562 \\
\hline Variovorax ginsengisoli Im et al. 2010 & sp. nov. & 1568 \\
\hline Labrys Vasilyeva and Semenov 1985 emend. Albert et al. 2010 & emend.* & 1575 \\
\hline Labrys wisconsinensis Albert et al. 2010 & sp. nov. & 1575 \\
\hline Winogradskyella Nedashkovskaya et al. 2005 emend. Ivanova et al. 2010 & emend.* & 1579 \\
\hline Winogradskyella exilis Ivanova et al. 2010 & sp. nov. & 1579 \\
\hline Luteimonas terricola Zhang et al. 2010 & sp. nov. & 1582 \\
\hline Flavihumibacter Zhang et al. 2010 & gen. nov. & 1611 \\
\hline Flavihumibacter petaseus Zhang et al. 2010 & sp. nov. & 1611 \\
\hline
\end{tabular}


cont.

\begin{tabular}{|c|c|c|}
\hline Name/author(s): & Proposed as: & Page no. \\
\hline Marinicella Romanenko et al. 2010 & gen. nov. & 1617 \\
\hline Marinicella litoralis Romanenko et al. 2010 & sp. nov. & 1617 \\
\hline Celeribacter Ivanova et al. 2010 & gen. nov. & 1623 \\
\hline Celeribacter neptunius Ivanova et al. 2010 & sp. nov. & 1623 \\
\hline Desulfopila inferna Gittel et al. 2010 & sp. nov. & 1629 \\
\hline Psychrobacter aestuarii Baik et al. 2010 & sp. nov. & 1633 \\
\hline Prevotella dentasini Takada et al. 2010 & sp. nov. & 1639 \\
\hline Dyadobacter psychrophilus Zhang et al. 2010 & sp. nov. & 1642 \\
\hline Muriicola Kahng et al. 2010 & gen. nov. & 1647 \\
\hline Muriicola jejuensis Kahng et al. 2010 & sp. nov. & 1647 \\
\hline Shewanella fodinae Sravan Kumar et al. 2010 & sp. nov. & 1652 \\
\hline Oceanicola nitratireducens Zheng et al. 2010 & sp. nov. & 1657 \\
\hline Actinaurispora Thawai et al. 2010 & gen. nov. & 1662 \\
\hline Actinaurispora siamensis Thawai et al. 2010 & sp. nov. & 1663 \\
\hline Tsukamurella soli Weon et al. 2010 & sp. nov. & 1670 \\
\hline Pseudoclavibacter chungangensis Cho et al. 2010 & sp. nov. & 1675 \\
\hline Actinomyces hominis Funke et al. 2010 & sp. nov. & 1679 \\
\hline Sphingopyxis soli Choi et al. 2010 & sp. nov. & 1684 \\
\hline Microbacterium azadirachtae Madhaiyan et al. 2010 & sp. nov. & 1690 \\
\hline Heliobacteriaceae Madigan and Asao 2010 & fam. nov. & 1709 \\
\hline
\end{tabular}

${ }^{\star}$ Taxonomic opinion. 